\title{
Toward 1000 Submissions in 2021: JPAH's First Trimester Report
}

\section{Pedro C. Hallal}

Over the first 3 months of 2021, the Journal of Physical Activity and Health (JPAH) received 224 submissions from 43 different countries. These numbers put the journal on pace to receive closer to 1000 submissions in 2021, as compared to 876 in 2020 and fewer than 700 in each of the previous five years. Given the high volume of submissions, JPAH will be inviting two new Senior Associate Editors over the next weeks. Another important activity JPAH will be undertaking in 2021 is a revamping of the Editorial Board. JPAH's priority to "go global" will be the underlying principle of the restructuring.

Of the 224 articles the journal received in the first trimester, $76(34.1 \%)$ came from North America, 51 (22.9\%) from Europe, $46(20.6 \%)$ from South or Central America, 37 (16.6\%) from Asia, $8(3.6 \%)$ from Oceania and $6(2.7 \%)$ from Africa. Increasing the number of submissions from Asia and Africa is a clear priority, as these regions comprise $59.5 \%$ and $17.2 \%$ of the world's population, respectively.

A closer look at the numbers reveals that 166 (74\%) of 224 submissions were rejected without peer review. The average time from submission to desk rejection was 1.1 days; $90 \%$ of these articles were rejected within 3 days from submission. Of the 58 articles that were sent to peer review, the average time from submission to first decision was 37 days.

There were 3 main reasons for desk rejections: (1) articles that are not within the scope of the journal, particularly those from exercise sciences that fail to directly connect with the field of physical activity and health; (2) articles that are methodologically flawed due to small sample size or inappropriate study design-in most cases, cross-sectional studies trying to address cause-effect associations; (3) review articles without a clear research question. In particular, the use of techniques to summarize the findings of existing articles, such as meta-analyses, are highly recommended in review articles. Narrative reviews will rarely be accepted. JPAH is aware that most graduate students need to conduct a review of the literature as part of their studies. However, these reviews do not automatically qualify as scientific articles.

In order to make sure editors are consistent in evaluating articles submitted to JPAH, a blinded experiment will take place over the coming months so that the agreement across editors can be quantified. For transparency, JPAH will share the results with its community.

It is impossible to write an editorial about JPAH's first trimester without mentioning the reviewers. On the one hand, it is getting harder and harder to secure reviewers-JPAH is currently having to invite 6 to 8 reviewers, on average, to secure a necessary 2 commitments. On the other hand, JPAH is getting excellent reviews from those who are available and, consequently, we have decided to acknowledge the work of superb reviewers toward the end of each calendar year. No further spoilers here.

Finally, researchers in the field should never forget that the quality of the work JPAH publishes is a consequence of the submissions we receive. Commitment from physical activity researchers around the globe to submit their most interesting articles to JPAH is essential for our continuous improvement. 